\title{
Pore space and specific surface area of heavy clay Oxisols as affected by their mineralogy and organic matter
}

\begin{abstract}
Pores and specific surface area (SSA) play a major role in controlling transport systems and potential reactivity of soils, but they have received little attention for heavy clay Oxisols. The objectives of this study were: (i) to study the pore sizes and SSA of heavy clay Oxisols as affected by their mineralogy and soil organic matter (SOM); and (ii) to determine the mechanism of SOM stabilization in a dark-colored Oxisol (Kuantan soil). The mineralogy of the clay fraction was studied by X-ray diffraction, differential thermal analysis, and scanning electron microscopy, whereas soil organic $\mathrm{C}$ was determined by combustion technique. Pores and SSA were measured by N2 adsorption-desorption technique. Results showed that the clay fraction of the three Oxisols studied varied from $73 \%$ to $82 \%$. The minerals in this soil fraction were kaolinite, goethite, hematite, and gibbsite in varying amounts. Under natural conditions (SOM was not removed by hydrogen peroxide), the pores were dominated by mesopores $(2-50 \mathrm{~nm})$. Partitioning the mesopores into various sizes showed that the cumulative small mesopore $(2-20 \mathrm{~nm})$ volume was considerably higher for Kuantan $(63 \%$ $73 \%)$ than the S. Mas or Segamat (25\%-35\%) soils. This is caused by the lower crystallinity and smaller size of minerals in the former. The values of SSA were compatible with those of the soil pores as exhibited by the SSA with and without SOM, which were 1.6 to 1.9 and 2.0 to 2.2 times higher, respectively, in Kuantan than in S. Mas or Segamat soils. Stabilization of SOM in Kuantan soil occurred through physical protection in the mesopores and cation bridging, as revealed by the increase in mesopore volume (from $58 \%$ to $92 \%$ ) after SOM removal and the high Fep and Alp contents, respectively. The less crystalline and smaller clay size particles present in the heavy clay Oxisols have a positive impact on soil carbon sequestration and stabilization.
\end{abstract}

Keyword: Oxisols; Surface area; Pore; Mineral, Organic matter 